\title{
Is an Isolated Weight-Holding Tremor a New Subtype of Isometric Tremor?
}

\author{
Seok Woo Yong ${ }^{1}$, Don Gueu Park ${ }^{1}$, Jung Han Yoon ${ }^{1}$, and Jong Sam Baik² \\ ${ }^{1}$ Department of Neurology, College of Medicine, Ajou University, Suwon; \\ ${ }^{2}$ Department of Neurology, Sanggye Paik Hospital, Inje University College of Medicine, Seoul, Korea.
}

\begin{abstract}
A weight-holding tremor is a rare type of hand tremor that occurs only when someone holds some weight. Here we report three cases of isolated weight-holding tremors, of which one patient was diagnosed with Parkinson's disease (PD) and the others as a variant of essential tremor (ET). A 68-year-old woman presented with a left-hand tremor that appeared only when she held objects with some weight. Her tremor was reminiscent of the re-emergent tremor of PD, and dopamine transporter imaging revealed reduced uptake at the right posterior putamen. A 21-year-old man and a 71-year-old woman also presented with similar weightholding tremors. However, these tremors were not re-emergent, and no signs of parkinsonism were observed during follow-up. Although the underlying etiologies of these tremors differed, all three tremors worsened as the held weight increased. These tremors could be isolated isometric tremors, but further research is needed to clarify the nature of this rare tremor.
\end{abstract}

Key Words: Parkinson's disease, tremor, weight-holding

\section{INTRODUCTION}

A weight-holding tremor is a rare type of hand tremor that occurs only when the patient holds some weight. Lang, et al. ${ }^{1}$ first described this tremor, suggesting that it was a variant of essential tremor (ET), and since then a few similar cases have been reported. The cause of this phenomenon remains unclear. We recently encountered three patients with isolated weight-holding tremors, and here we discussed the nature of this rare motor phenomenon.
Received: March 24, 2020 Revised: May 13, 2020

Accepted: May 29, 2020

Corresponding author: Jong Sam Baik, MD, PhD, Department of Neurology, Sanggye Paik Hospital, Inje University College of Medicine, 1342 Dongil-ro, Nowon-gu, Seoul 01757, Korea.

Tel: 82-2-950-1090, Fax: 82-2-950-1955, E-mail: jsbaik@paik.ac.kr

-The authors have no potential conflicts of interest to disclose.

(C) Copyright: Yonsei University College of Medicine 2020

This is an Open Access article distributed under the terms of the Creative Commons Attribution Non-Commercial License (https://creativecommons.org/licenses/ by-nc/4.0) which permits unrestricted non-commercial use, distribution, and reproduction in any medium, provided the original work is properly cited.

\section{CASE REPORTS}

\section{Case 1}

A 68-year-old woman presented with a left-hand tremor that had become progressively worse over the previous 12 months. She was a housekeeper, was right-handed, and first noticed the tremor while carrying objects around the house. None of her relatives were known to have ET or Parkinson's disease (PD), and she denied taking any gastrokinetic, antipsychotic, or antiepileptic drugs; her only medication was amlodipine $5 \mathrm{mg}$ per day for high blood pressure. On neurological examination, she showed no signs of resting tremor, postural tremor with an outstretched posture, or kinetic tremor during the finger-to-nose maneuver. The tremor was absent when she held an empty can with her left hand, but it appeared when she held a full can (Supplementary Video Segment 1, only online). The tremor was reminiscent of a re-emergent tremor in that it had a latency of 2 to 4 seconds and a frequency of $5 \mathrm{~Hz}^{2}$ The patient showed no signs of suggestibility or distractibility. We confirmed the absence of resting or postural tremors or a tremor when holding an empty can by electromyography (EMG) of her supinator and pronator teres muscles; alternating $5-\mathrm{Hz}$ discharges were visible only when she was holding a full can (Fig. 1A). The patient had no rigidity, bradykinesia, or postural instability. Her mental and cranial nerve functions and gait were all normal. Brain 


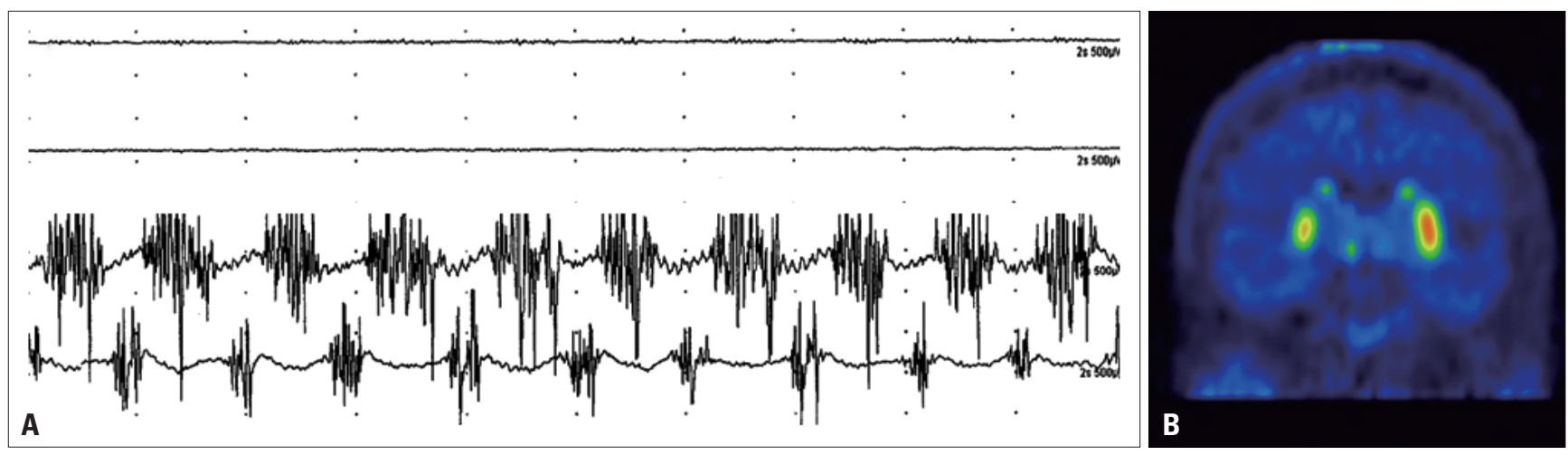

Fig. 1. (A) EMG recording of the left-hand supinator and pronator teres. The upper two traces were taken when the patient was holding an empty can, and the lower two while she was holding a filled can. (B) Coronal ${ }^{18} \mathrm{~F}-\mathrm{FP}$-CIT PET section showed reduced uptake in the dorsal putamen, which was more pronounced on the right.

magnetic resonance imaging (MRI) was unremarkable. $\mathrm{N}-(3-$

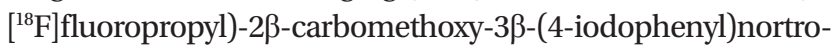
pane $\left({ }^{18} \mathrm{~F}-\mathrm{FP}-\mathrm{CIT}\right)$ positron emission tomography revealed reduced uptake in the posterior-dorsal putamen, which was more pronounced on the right (Fig. 1B). She began to develop very mild signs of bradykinesia and a resting tremor after 10 months of follow-up. We started treatment with levodopa $200 \mathrm{mg}$ per day, but her tremor did not improve.

\section{Case 2}

A 21-year-old man presented with bilateral hand tremors for 1 year. He was right-handed, and had first noticed the tremor during army duty when completing various manual tasks, such as shooting and carrying objects. He told us that the tremor was worse when he carried heavy objects in his hand. He had no family history of ET or PD, and denied taking any medications. On neurological examination, he had no resting tremor or kinetic tremor during the finger-to-nose maneuver, but had a mild symmetric postural tremor in an outstretched position. He had no visible tremor when holding an empty bag, but the tremor appeared without any latency when a book was placed in the bag. The amplitude of the tremor increased with the weight held. In entrainment test, there was no distractibility nor entrainment of tremor frequency. There was no rigidity, bradykinesia, or postural instability. Laboratory findings were all normal, including serum ceruloplasmin and thyroid hormone. Brain and cervical spine MRI were unremarkable. EMG and FP CIT-PET were not performed. After taking propranolol $40 \mathrm{mg}$ per day, his tremor improved markedly.

\section{Case 3}

A 71-year-old women presented with a left-hand tremor for 4 years. The tremor appeared only when she was holding objects with her first and second fingers, and the tremor amplitude tended to increase when she held heavier objects (Supplementary Video Segment 2, only online). There was no evident resting, postural, or other kinetic tremor. No signs of parkinsonism were observed, and there was no dystonic posturing in any of her limbs. She denied any progression of symptoms during the last 4 years, after she first noticed the symptoms. She had no family history of ET or PD, and the only medication she was taking was tamoxifen for invasive ductal carcinoma in her right breast, which she had started 3 years previously. We prescribed propranolol $40 \mathrm{mg}$ per day and trihexyphenidyl $1 \mathrm{mg}$ per day, but her tremor did not improve. She refused to undergo ${ }^{18} \mathrm{~F}-\mathrm{FP}$ CIT PET. No signs of parkinsonism were observed at the 1-year follow-up.

\section{DISCUSSION}

All three patients had similar isolated weight-holding tremors. Considering the phenomenology of their tremors, the first tremor and the other two had different patterns: the first patient had a re-emerging tremor pattern, while the other two had tremors that increased with heavier weight. The second and third cases were similar to that reported by Lang, et al. ${ }^{1}$ Subsequently, we found that both patients had possible ET. In case 3, we could not completely rule out the possibility of parkinsonian tremor due to the lack of dopamine transporter imaging; however, the absence of symptom progression in the last 4 years and additional 1 year of follow-up made PD less likely. Interestingly, we found some unique patterns in the three patients. The first had no resting tremor, but had a unilateral tremor that resembled the re-emerging tremor in patients with PD. This unique tremor pattern prompted us to investigate possible $\mathrm{PD}^{2}{ }^{2}$ There have been some reports of cases with PD with a re-emerging tremor but no resting tremor. ${ }^{3}$ Unlike the previously reported cases, the amplitudes of tremors in our patients increased with the weight load, suggesting that weight loading has little effect on postural hand tremors in patients with PD. ${ }^{4}$

Since the first description of a weight-bearing tremor by Lang, et al., ${ }^{1}$ there have been reports of similar cases; these might be a form of isolated isometric tremor. ${ }^{5,6}$ Similar to our second and third cases, these reports described unilateral or bilateral tremors appearing almost exclusively with weight-holding tasks. 
Although these cases did not meet the criteria for PD, ET, or other tremor syndromes, ${ }^{7}$ the frequency of the tremors was similar to that of PD. Moreover, in all of these cases, including ours, placing a weight on the hand exacerbated the tremor, which is opposite to what is commonly observed in patients with ET. Therefore, these cases suggest an unrecognized subtype of isometric tremor or a variant of ET.

These tremors may resemble task-specific tremor, if weightbearing could be considered a "specific task." However, as discussed in earlier reports, task specificity requires skilled nature of the task, which often involves the use of a specific instrument or tool. ${ }^{5,8}$ In our cases, holding any object with suitable weight elicited the tremor, which in our opinion, made it different from a task-specific tremor.

While weight-holding tremors are rare, the underlying etiology might be diverse. Based on our cases, we add the important phenomenology of a re-emerging tremor pattern to the diagnosis of PD. The opposite phenomenon-an isometric tremor or a variant of ET that increases in amplitude with an increasing load-suggests another subtype of isometric tremor. More research and similar cases are needed to characterize these difficult-to-classify tremors.

\section{SUPPLEMENTARY DATA}

Video Segment 1. The patient had a tremor of the left hand only when lifting a filled can or bottle, with a tremor emergence latency of 2-4 seconds (shown twice). Segment 2 . The left-hand tremor appeared as the patient held a pen, and was aggravated when she held a heavier object. No postural tremor was observed without weight-holding.

The patients gave informed consent to be videoed for publication.

\section{ACKNOWLEDGEMENTS}

The first author, Seok Woo Yong, who was a professor, colleague, friend, and father of Hyun Jin Yong, passed away on June 13, 2013. His spirit and uncompromising enthusiasm for move- ment disorders will never be forgotten and will always remain an inspiration.

\section{AUTHOR CONTRIBUTIONS}

Conceptualization: Seok Woo Yong and Jong Sam Baik. Investigation: Seok Woo Yong. Project administration: Jung Han Yoon and Jong Sam Baik. Resources: Seok Woo Yong and Don Gueu Park. Supervision: Jong Sam Baik. Visualization: Seok Woo Yong. Writing—original draft: Seok Woo Yong and Don Gueu Park. Writing_review \& editing: Jung Han Yoon and Jong Sam Baik. Approval of final manuscript: all authors.

\section{ORCID iDs}

Don Gueu Park Jung Han Yoon Jong Sam Baik

https://orcid.org/0000-0001-8658-3523 https://orcid.org/0000-0001-6180-9848 https://orcid.org/0000-0002-5300-203X

\section{REFERENCES}

1. Lang AE, Jog M, Ashby P. "Weight-holding tremor": an unusual task-specific form of essential tremor?. Mov Disord 1995;10:228-9.

2. Jankovic J, Schwartz KS, Ondo W. Re-emergent tremor of Parkinson's disease. J Neurol Neurosurg Psychiatry 1999;67:646-50.

3. Louis ED, Pullman SL, Eidelberg D, Dhawan V. Re-emergent tremor without accompanying rest tremor in Parkinson's disease. Can J Neurol Sci 2008;35:513-5.

4. Meshack RP, Norman KE. A randomized controlled trial of the effects of weights on amplitude and frequency of postural hand tremor in people with Parkinson's disease. Clin Rehabil 2002;16: 481-92.

5. Robakis D, Louis ED. Another case of "shopping bag" tremor: a difficult to classify action tremor. Tremor Other Hyperkinet Mov (NY) 2014;4:269.

6. Zesiewicz T, Vu T, Carranza MA, Appelbaum R, Snyder M, Staffetti JS, et al. Unusual wrist tremor: unilateral isometric tremor?. Tremor Other Hyperkinet Mov (N Y) 2014;4:tre-04-194-4738-1.

7. Bhatia KP, Bain P, Bajaj N, Elble RJ, Hallett M, Louis ED, et al. Consensus statement on the classification of tremors. from the task force on tremor of the International Parkinson and Movement Disorder Society. Mov Disord 2018;33:75-87.

8. Bain PG. Task-specific tremor. Handb Clin Neurol 2011;100:711-8. 